\title{
La misión de las bibliotecas públicas en la nueva ecología informacional: una perspectiva hispanoamericana
}

\author{
Francisco Javier García Marco \\ Universidad de Zaragoza (España)
}

\section{Resumen}

Se analizan los cambios que se están produciendo en el mundo de las bibliotecas públicas al hilo de la presentación de los artículos que componen el número monográfico de la revista Scire: Organización y Representación del Conocimiento (vol. 1, n. ${ }^{\circ}$ 1, en.-jun. 2007). En primer lugar, se analiza la misión de la biblioteca pública al servicio del desarrollo social, a través del apoyo a la educación y el autoaprendizaje, abordando los cambios que se están produciendo en el acceso a la información y el impacto de las desigualdades sociales dentro y entre los países. Al facilitar el aprendizaje, y especialmente el autoaprendizaje, la biblioteca pública sigue siendo, sobre todo, un instrumento de educación y promoción social para todos. En segundo lugar, se discute la incorporación de la biblioteca pública al universo digital, que se ha convertido en parte ineludible de la "cultura material" contemporánea. El entorno de cambio tecnológico exige una realineación de tareas y funciones entre los diferentes subsistemas de la "ecología informacional" contemporánea. Algunas de las tareas para las que la biblioteca pública aparece especialmente bien posicionada son preservar y difundir el patrimonio local, ampliar su servicio de proximidad a otros tipos de información y asistir a los ciudadanos en el acceso a la información digital.

Palabras clave: Bibliotecas públicas. Latinoamérica. España. Modelos de gestión. Estudios de usuarios. Uso de la información. Recursos electrónicos.

\section{Abstract}

The current changes in the world of public libraries are examined, as the articles published in the current monographic of the journal Scire (vol. 13, no. 1, Jan.-June 2007) are commented. First, the mission of the public library at the service of social development is examined. This service is made possible by facilitating learning, and mainly self-learning. So, the library must be considered an educational tool for social promotion and personal self-actualization. Second, the incorporation of public libraries to the digital arena is discussed. The Internet 
requires a new configuration of the public library services into the emergent "information ecology". Some of the tasks in which the public library may excel are assisting citizens in accessing and navigating the digital universe, preserving and disseminating the local information heritage, and expanding its proximity service factor into a wider conception of citizen information.

Keywords: Public libraries. Latin America. Spain. Management models. User studies. Information use. Electronic resources.

\section{Introducción}

Este primer número del volumen 13 de la revista Scire: Organización y Representación del Conocimiento (en.-jun. 2007) recoge artículos dedicados monográficamente a las bibliotecas públicas y su evolución en la era de la red Internet.

Los trabajos publicados abordan fundamentalmente dos temas: en primer lugar, la misión de la biblioteca pública al servicio del desarrollo social, entendido de la mejor manera, esto es, como desarrollo de las personas que componen las sociedades; en segundo lugar, la incorporación de la biblioteca pública al universo digital, que, lejos ya de ser una novedad tecnológica, se está convirtiendo en la infraestructura comunicacional de la sociedad contemporánea, incorporándose plenamente a lo que algunos científicos sociales denominan cultura material. En relación con este segundo frente, también se aborda la cuestión del impacto del cambio tecnológico sobre los servicios de las bibliotecas públicas, en un entorno que exige una realineación de tareas y funciones entre los diferentes subsistemas de la "ecología informacional" actual.

\section{La biblioteca pública al servicio del desarrollo social}

Podemos aceptar la Public Libraries Act de 1850 como el inicio formal de las bibliotecas públicas actuales, con la misión de abrir el mundo de la lectura a todos los ciudadanos. Uno de sus tres promotores, Edward Edwards, era un bibliotecario, antiguo albañil, que en 1839 llegó a ser asistente en el Departamento de Libros Impresos del Museo Británico, habiéndose formado a sí mismo en sus ratos libres leyendo en las bibliotecas del Mechanics' Institute. Los otros dos promotores de la ley fueron Wiliam Ewart y Joseph Brotherton, dos miembros liberales del parlamento británico. Esta significativa anécdota contiene muchas de las connotaciones del concepto actual de biblioteca pública: instrumento de promoción social a través de la autoeducación que permite la lectura y que presupone, lógicamente, el acceso a los libros, los periódicos y las revistas; y una concepción de lo público democrática, es decir, extensiva a todos los miembros de la sociedad, sin elitismos ni exclusiones.

El ideal de biblioteca pública es realmente muy alto, y no es extraño que su consecución esté permanentemente amenazada por factores tanto externos como

Scire. $13: 1$ (en.-jun. 2007) 9-19. ISSN 1135-3716. 
internos. Entre las amenazas intrínsecas que acechan constantemente a la misión de la biblioteca pública destaca la de quedarse en un simple almacén, mejor o peor organizado, de documentos, ya que la biblioteca pública es mucho más, si atendemos a su función y a su historia. En esta dirección abunda precisamente el sólido y transcendente estudio de Emir José Suaiden (2007) sobre la dimensión social del conocimiento que abre el monográfico.

Para Suaiden, esta dimensión social se puede entender como "un nivel de conciencia colectiva que exige la mejor distribución de los saberes y de las riquezas generadas por la sociedad". El autor aborda la misión de la biblioteca pública en la sociedad del conocimiento y define las sociedades del conocimiento como aquellas sociedades que han conseguido organizarse para generar y usar el conocimiento de forma sistemática, contribuyendo al proceso de aprendizaje social y, en definitiva, a construir un futuro mejor. Coincide con Castells (1994, p. 25, y 1997, p. 103) en que en las sociedades del conocimiento, la productividad, el crecimiento económico, la riqueza y el poder descansan en el control del conocimiento y de la información, y especialmente, podríamos añadir, en el control de su difusión social. Pues bien, Suaiden — desde su perspectiva comprometida con el desarrollo de América Latina- enfatiza la necesidad de un sistema educativo en un sentido amplio que facilite el acceso a la información y al conocimiento de toda la población. Así, América Latina podrá desarrollar conocimientos propios que se reflejen en una producción editorial importante y en la obtención de patentes, que ahora importa en su gran mayoría de fuera. Solo así se podrá conseguir un desarrollo armónico y sostenible.

Pues bien, un elemento clave de ese sistema educativo debe ser la biblioteca, "que puede transformar la calidad de vida de las comunidades diseminando información adecuada con la rapidez que los tiempos modernos exigen". Para el autor, "la biblioteca debería ser el gran instrumento de educación, especialmente en lo que respecta a la dimensión social del conocimiento", a la lucha contra la exclusión en el plano del conocimiento. Pero, para ello, la actividad bibliotecaria debe trascender la colección para convertirse en un puente entre esta y las personas que se pueden beneficiar de los conocimientos pero que son incapaces de hacerlo por desmotivación, desconocimiento o deficiencias educativas. Se trata, en definitiva, de abordar sin demora lo que el autor denomina la "dimensión humana de la información". Para ello, Emir Suaiden no duda en denunciar un modelo de biblioteca "invisible" que "no provoca ningún impacto social en las organizaciones que teóricamente tendrían que actuar como propagadoras y multiplicadoras de una política educacional, cultural y social como el sistema educacional".

En otro artículo, María de los Ángeles Ciprés Oliva y Alva Flores Romero (2007a) abordan una cuestión bibliotecaria también eminentemente social: las prioridades de gasto en las redes de bibliotecas públicas. En concreto, las autoras 
contrastan el proyecto de la megabiblioteca José Vasconcelos de la Ciudad de México — destinada a ser la cabecera de la red nacional de bibliotecas públicas y su centro de referencia - con el grado de desarrollo de la red de bibliotecas públicas de las delegaciones, que estudian mediante la literatura profesional, la información publicada en los medios, cuestionarios y la observación directa. Las encuestas abordan aspectos tan interesantes como la opinión de los bibliotecarios ante el proyecto - que lo apoyan en su tercera parte—, a la vez que desgranan detalladamente el estado de la red bibliotecaria de la megalópolis mexicana. Ante la situación de las bibliotecas de la red, las autoras cuestionan abiertamente la conveniencia del proyecto y recomiendan que el Consejo Nacional para la Cultura y las Artes (Conaculta) establezca sus prioridades de acuerdo con las necesidades reales de la Red Nacional de Bibliotecas Públicas, establecidas, por otra parte, en el diagnóstico y los planes de trabajo correspondientes a dicha Red, ya presentados en el Programa Nacional de Cultura 2001-2006. En cualquier caso, las autoras establecen una metodología y un estudio de referencia para abordar un conflicto de carácter universal en las políticas culturales: las grandes infraestructuras de referencia ejemplificadoras del poder del Estado — o más bien de las personas y las élites que lo controlan y que se identifican con él- frente al desarrollo de infraestructuras desde la base y cercanas a las comunidades a las que se pretende servir.

En su segundo trabajo, María de los Ángeles Ciprés Oliva y Alva Flores Romero (2007b) afrontan un problema específico que planteaba Emir Suaiden en su artículo sobre la misión social de la biblioteca pública, esto es, cómo acercar el conocimiento custodiado en las bibliotecas a un público que, por deficiencias formativas y actitud ante la vida, puede encontrarse, en muchas ocasiones, alejado de ellas. Lo abordan para un segmento de la población especialmente abierto al saber - y necesitado de él— por encontrarse en su etapa formativa: la población infantil. De nuevo se trata de un estudio desarrollado en la Red de Bibliotecas Públicas de la Ciudad de México por medio de encuestas y apoyado en el análisis de la literatura previa. Las autoras prueban y constatan para el caso concreto de la Ciudad de México el problema de alcance general que Emir Suaiden presentaba en su artículo: en el común de las bibliotecas, los esfuerzos proactivos de motivación son muy escasos y, aun en los casos en los que existen efectivamente, suelen ser en su gran mayoría deficientes.

Las autoras analizan con detalle las líneas de trabajo y las dinámicas empleadas. La lectura de cuentos es la actividad principal de promoción de la lectura, aunque se llevan a cabo otras acciones como talleres, manualidades, visitas guiadas, juegos, asesoría de tareas, círculos de lectura, dibujo, teatro y otros. Sin embargo, las actividades se realizan de forma muy esporádica y generalmente en verano, de modo que, en general, la biblioteca queda convertida en un mero instrumento de apoyo a la actividad educativa durante gran parte del año. Las autoras entre- 
vistaron también a niños para obtener un panorama complementario, que, en su conjunto, convierte a este trabajo en un estudio de referencia por la metodología utilizada y la claridad de sus resultados y recomendaciones.

En un ámbito más específico, Margarita Pérez Pulido y José Luis Herrera Morillas (2007) abordan también la dimensión social de la biblioteca pública; en este caso, a través del papel realizado por este tipo de centros en las prisiones. Los autores clarifican el marco de actuación de estos servicios a partir del análisis de las normas y recomendaciones internacionales. Así, la biblioteca de la prisión se concibe como un instrumento de "apoyo a las actividades educativas del centro, al desarrollo personal y cultural de los reclusos, a su formación para el empleo y a la ocupación de sus momentos de ocio y distracción”, cuyos aspectos técnicos deben estar a la altura de las exigencias de una biblioteca pública. Los autores analizan las experiencias y modelos estadounidense, británico y español, y concluyen proponiendo un nuevo modelo organizativo para el caso español, que pasa necesariamente "por su integración de manera formal en los sistemas bibliotecarios de las comunidades autónomas".

\section{El cambio digital y los nuevos servicios en las bibliotecas públicas}

Una cuestión fundamental para los servicios de información y documentación en general y las bibliotecas públicas en particular es la intensa reconfiguración que están sufriendo estas áreas de la actividad social como consecuencia del impacto de las tecnologías de la información y la comunicación (TIC). En un trabajo anterior (García, 1998) examinábamos cómo la automatización creciente de las tareas bibliotecarias y el aprovechamiento de los recursos primarios y secundarios a través de Internet estaba minimizando rápidamente la dedicación a las tareas que tradicionalmente han ocupado más tiempo en las bibliotecas de nuestros países - los procesos técnicos-, a la vez que desplazaba el énfasis hacia servicios más desatendidos — como la referencia, la formación de usuarios o la "extensión"pero más cercanos a los fines de la biblioteca, y promovía una "relocalización" de las funciones de las bibliotecas, más ligadas a su entorno local para ofrecerlo a través de Internet. Esa tendencia no ha hecho sino incrementarse durante toda la década que ahora está terminando, durante la cual la competencia con servicios "globalizados" emergentes no ha hecho sino crecer: Google Books, Google Scholar, Amazon, iTunes, etcétera.

Este monográfico contiene varios trabajos que abordan precisamente algunas de las respuestas que se están produciendo desde las bibliotecas públicas y, en particular, muestran el desarrollo de las "nuevas" funciones bibliotecarias que se ofrecen como respuesta a la realineación profunda que se está produciendo en el mundo de la información y la comunicación como consecuencia del impacto de la revolución teleinformática.

Scire. $13: 1$ (en.-jun. 2007) 9-19. ISSN 1135-3716. 
Tanto el trabajo de José Luis Herrera Morillas (2007) como el de Isabel Andreu Felipe, Carmen María Brugarolas Ros, Concepción Dolores Alcázar Ruiz y José Luis Cárceles Andreu (2007) plantean desde diferentes perspectivas el problema del posicionamiento de las bibliotecas públicas en el entorno digital.

El primero de ellos aborda "La imagen corporativa de las bibliotecas autonómicas españolas a través de Internet". Se trata de un trabajo interesante y novedoso que estudia la presencia en Internet de las bibliotecas autonómicas españolas, y que además lo hace desde el punto de vista de su imagen corporativa. En el momento de la realización del estudio contaban con sitio web la Biblioteca de Andalucía, la Biblioteca de Aragón, la Biblioteca Central de Cantabria, la Biblioteca de Castilla y León, la Biblioteca de Castilla - La Mancha, la Biblioteca Nacional de Catalunya, la Biblioteca Valenciana, la Biblioteca Central de La Rioja, la Biblioteca Regional de Murcia, la Biblioteca General de Navarra y el Centro Superior Bibliográfico de Galicia. El artículo muestra el esfuerzo que han hecho las bibliotecas autonómicas por ofrecer una imagen corporativa elaborada en Internet, que resulta hoy en día imprescindible para cualquier organización seria.

En el segundo artículo que nos interesa ahora, Isabel Andreu Felipe, Carmen María Brugarolas Ros, Concepción Dolores Alcázar Ruiz y José Luis Cárceles Andreu (2007,) estudian las "Aplicaciones de los blogs en unidades de información: usos y perspectivas". En la década a cuyo fin nos aproximamos, los blogs han irrumpido como un nuevo medio de comunicación que compite por el favor del público con los grandes medios, que a su vez están intentando integrarlo en sus estructuras reorganizando sus empresas digitales bajo la forma de portales especializados temática o ideológicamente. De alguna manera, el fenómeno blog da cumplimiento a la promesa de Internet como plataforma capaz de democratizar no solo el acceso a la información, sino, sobre todo, su publicación. Pues bien, en este trabajo los autores repasan el concepto de blog, las tecnologías que lo soportan y, especialmente, sus usos y aplicaciones más frecuentes en las unidades de información, de los que ofrecen diversos ejemplos prácticos. Los blogs resultan útiles en referencia, en marketing, en información de actualidad y en crítica de libros y artículos, entre otras posibilidades, y de una manera especial para dinamizar la participación de los usuarios. Su uso no ha parado de crecer y será cada vez más importante en las bibliotecas, archivos y centros de documentación de referencia.

Ciertamente, como muestran estos estudios, el futuro de la biblioteca pública no pasa solo por dar la publicidad a la marca y los servicios de las bibliotecas públicas aprovechando las nuevas tecnologías de la información y la comunicación. Sobre todo se trata de ofrecer un producto diferenciado para públicos segmentados con criterio; diferenciado, en primer lugar, frente a otros tipos de servicios de información y documentación emergentes, especialmente en el mundo digital, pero también frente a otras bibliotecas, que cuentan muchas veces con mayores recur- 
sos y que están extendiendo su público fuera de sus fronteras tradicionales gracias al acceso por Internet y las colecciones y servicios digitales. Un riesgo emergente - al competir en un entorno globalizado gracias a Internet mientras que una parte creciente de sus fondos van estando disponibles en formato digital en la Red-es el de que las bibliotecas públicas puedan ir perdiendo su papel casi monopolístico como servicio de proximidad y sin competencia en su distrito "físico". Es necesario reforzar las ventajas competitivas que les proporciona ser un servicio de proximidad, que tiene realidad física además de virtual.

Otro grupo de artículos incide precisamente en diferentes funciones que las bibliotecas públicas pueden potenciar en este nuevo contexto para maximizar su ventaja competitiva. Se trata de la formación de usuarios, la preservación y difusión del patrimonio local y la ampliación de los servicios de proximidad para llegar a constituir una especie de ventanilla única de información ciudadana.

En primer lugar, el artículo de María Sánchez Hernández (2007) sobre "El acceso y la difusión de los recursos bibliográficos e informativos en la Biblioteca Regional de Extremadura" incide en las funciones de preservación y difusión del patrimonio documental local, que la autora ejemplifica analizando las funciones, servicios y actividades de este centro. La misión de la Biblioteca Regional de Extremadura es asegurar la accesibilidad a la información para la sociedad extremeña, tanto con las fuentes documentales que en ella se conservan como las depositadas en otras bibliotecas. Para ello, la Biblioteca debe garantizar la recogida, conservación y difusión del patrimonio informativo de la comunidad autónoma en cualquier soporte, actividades que se hacen especialmente visibles a través de dos publicaciones: la Bibliografía regional —en curso y retrospectiva- y el Boletín del Depósito Legal de Extremadura. El artículo presta una especial atención a un proyecto especialmente relacionado con la preservación y puesta en valor del patrimonio cultural extremeño: la organización del archivo fotográfico donado a la Biblioteca por El Periódico Extremadura. Está claro que las actividades de las bibliotecas regionales y locales en la preservación y difusión del patrimonio documental de sus jurisdicciones constituyen precisamente una de las líneas de futuro de estas instituciones, aunque, como se ha visto en Estados Unidos con Google, grandes empresas pueden estar interesadas también en competir en este ámbito.

Por otra parte, el trabajo de José Luis Herrera Morillas y Margarita Pérez Pulido (2007) aborda un horizonte importante para las bibliotecas públicas: llegar a convertirse en un punto de información o ventanilla única de información al ciudadano en colaboración con una Administración cada vez más sistematizada gracias a las nuevas tecnologías de la información y la comunicación. Su estudio es, sobre todo, de prospección y armonización teórica; para ello, analizan el concepto de información local en siete documentos de referencia sobre las bibliotecas

Scire. $13: 1$ (en.-jun. 2007) 9-19. ISSN 1135-3716. 
públicas: el Manifiesto de la Unesco (1994), la Declaración de Copenhague (1999), La biblioteca pública: un centro para la sociedad de la información (2000), las Directrices IFLA/Unesco (2001), las Pautas sobre los servicios de las bibliotecas públicas (2002), las Pautas Pulman (2002) y el Manifiesto de Oeiras (2003). Los autores resumen así sus conclusiones:

La biblioteca pública local se va configurando de este modo como un centro especializado en información local, mejor que otros tipos de bibliotecas (autonómicas, nacionales, etcétera) que, por tener unas funciones y tareas a veces demasiado ambiciosas, no están en condiciones de recopilar y conservar con exhaustividad toda la información y la documentación relacionada con una determinada localidad. Esta realidad se manifiesta de modo muy evidente en el caso de los materiales menores (folletos, catálogos, carteles, tarjetas, etcétera), pues una biblioteca nacional, e incluso una biblioteca autonómica, se puede ver fácilmente desbordada a la hora de controlar estos documentos. La exhaustividad y mayor cobertura de las colecciones y recursos relacionados con la localidad son los que realmente pueden dar una seña de identidad propia a las bibliotecas públicas de ámbito local frente a otros tipos de bibliotecas (Fornas, 2002, pp. 56-57).

\section{Conclusiones}

Las raíces y la misión de la biblioteca pública son asegurar el acceso a la cultura impresa —entendiendo el concepto de cultura en un sentido amplio, esto es, incluyendo también la cultura popular - para todos los ciudadanos, puesto que ese acceso facilita su educación a través del autoaprendizaje, y, en definitiva, les ayuda a realizarse como personas en sus diferentes aspectos y a ser más felices, rodeados a su vez de otras personas más realizadas y felices. Estas son sus raíces, su misión, su contexto..., y este es también su futuro: asegurar el acceso universal al saber almacenado en documentos públicos para promover la felicidad — por usar un término clásico- general.

Así pues, la biblioteca pública es un elemento fundamental de la red de socialización del conocimiento, en el sentido de que hace llegar el conocimiento a todo el que lo necesita, misión que comparte con la educación, la edición y los medios de comunicación. En el cumplimiento de este objetivo, su tarea no es solo pasiva - mantener colecciones-, sino también proactiva; esto es, ha de convertirse, a través de la acción y la reflexión de bibliotecarios comprometidos, en un puente entre el saber documentado y los ciudadanos. Y solo ese servicio garantizará su supervivencia, porque en él reside la razón de su existencia. De forma secundaria, pero también importante, la proactividad potenciará su éxito y extenderá su servicio, $\mathrm{y}$, proporcionalmente, su influencia.

Ahora bien, la sociedad del siglo XXI no es ya la del XX, y mucho menos la del XIX. La misión de la biblioteca tiene que realizarse en un nuevo marco, que se aparta del anterior en cuestiones fundamentales.

Scire. $13: 1$ (en.-jun. 2007) 9-19. ISSN 1135-3716. 
En primer lugar, la sociedad globalizada es cada vez más una sociedad de personas libres, con perspectivas, intereses y aficiones diferentes, segmentada a efectos de cualquier análisis en multitud de categorías multidimensionales. Hace falta desarrollar un nuevo sentido de lo público que contemple la diversidad sin caer en colectivismos simplificadores. Como muestran los trabajos de este monográfico, ser fieles a la misión de la biblioteca pública supone atender a todos desde el sistema público de bibliotecas — hemos visto el ejemplo de las bibliotecas de prisiones, con énfasis en las comunidades desfavorecidas (Suaiden, 2007; Ciprés y Flores, 2007a; Pérez y Herrera, 2007)—, y especialmente, dentro de ellas, a la población en formación (Ciprés y Flores, 2007b). Pero debilitaría la misión de la biblioteca pública el adoptar estas prioridades de forma exclusiva, dejando fuera a grandes sectores de la población. Entre los ciudadanos cultos y de situación desahogada, la biblioteca pública también puede aportar eficiencia en el uso de los recursos, en la tradición, por otra parte, de los clubs de lectura anglosajones, en los que la biblioteca pública tiene una de sus raíces.

En segundo lugar, aunque ciertamente no en todos los casos de la misma manera, la disponibilidad y el costo del acceso a la información han cambiado de forma radical en los últimos quince años. Hasta el último cuarto del siglo pasado la humanidad ha vivido en una sociedad pobre en información, donde acceder a los documentos era caro y penoso y en la cual existían grandes diferencias sociales en cuanto a las posibilidades de acceso a la misma. Internet ha dado un vuelco — todo lo incipiente que se quiera - a la situación. Un ejemplo paradigmático de los tiempos pasados frente a los nuevos puede ser la diferencia entre la Enciclopedia británica y la Wikipedia.

En estos momentos vivimos en una sociedad rica — isaturada? - en documentación, y la misión de la biblioteca pública tiene que adaptarse a este contexto. La referencia cobra de nuevo una importancia clave; una referencia que, por la propia naturaleza de los nuevos tiempos, tiene que ser colaborativa y en red, en el sentido que muestran servicios como Pregunte: las bibliotecas responden (http://www.pregunte.es/). Evaluar, cartografiar y guiar en los nuevos espacios de la información se ha convertido en un reto ineludible para la biblioteca pública, que, entre otras funciones, tendrá la de construir un centro de servicios de navegación para el ciudadano en sus viajes por el océano en que se ha convertido el mundo de la información y la documentación.

En tercer lugar, en un mundo cada vez más integrado — "globalizado"—, las actividades tradicionales de gestión de la información en las bibliotecas públicas estarán más sujetas a la especialización, la integración vertical y horizontal y la competencia mutua.

Mientras que los bibliotecarios dedican cada vez menos tiempo a adquirir y a catalogar, y sus bibliotecas se tecnologizan a un ritmo acelerado, las bibliotecas Scire. $13: 1$ (en.-jun. 2007) 9-19. ISSN 1135-3716. 
públicas encuentran un horizonte sólido y especializado en la combinación de dos líneas de actuación: por un lado, la preservación y difusión de la información local hacia el resto del mundo; por el otro, la apertura al ciudadano local de los recursos de un mundo globalizado, que, ahora sí, le conciernen, le afectan y le pueden ser útiles. Es una vertiente más de la omnipresente dinámica global/local, que constituye uno de los signos de nuestros tiempos. En ella la biblioteca pública reencuentra también su carácter mediador como interfaz humana entre la Red y los ciudadanos del distrito en el que actúa.

En fin, la biblioteca pública tiene un brillante futuro por delante en el cumplimiento de su misión de acercar la cultura a toda la población más allá de sus posibilidades económicas, aportando también una eficiencia sinérgica en sociedades ricas, pero si - y solo si - abraza con decisión — aunque sin exclusividad ni sobreactuaciones - las nuevas tecnologías de la información y la comunicación. Y es que, a estas alturas, las TIC, lejos de ser solo un interesante y apasionante avance tecnológico, forman ya parte de la cultura material de nuestras sociedades. En definitiva, la biblioteca pública o será en la Red o no será.

\section{Referencias}

Andreu Felipe, Isabel; Brugarolas Ros, Carmen María; Alcázar Ruiz, Concepción Dolores; Cárceles Andreu, José Luis (2007). Aplicaciones de los blogs en unidades de información: usos y perspectivas. // Scire: Representación y Organización del Conocimiento. 13:1 (en.-jun. 2007) 133-144.

Castells, Manuel (1994). Flujos, redes e identidades: una teoría crítica de la sociedad informacional. // Nuevas perspectivas críticas en educación. Actas del congreso internacional. Barcelona: Paidós, 1994. 13-54.

Castells, Manuel (1997). The power of identity: the information age: economy, society and culture. Vol. 2. Oxford: Blackwell, 1997.

Ciprés Oliva, María de los Ángeles; Flores Romero, Alva (2007a). La megabiblioteca José Vasconcelos y la situación de las bibliotecas públicas de la Ciudad de México. // Scire: Representación y Organización del Conocimiento. 13:1 (en.-jun. 2007) 51-66.

Ciprés Oliva, María de los Ángeles; Flores Romero, Alva (2007b). La motivación de la población infantil para lograr su asistencia a las bibliotecas públicas de la Ciudad de México. // Scire: Representación y Organización del Conocimiento. 13:1 (en.jun. 2007) $87-119$.

Fornas Carrasco, Ricardo (2002). La biblioteca pública municipal y los servicios de información local. // Métodos de Información. 51: 9 (2002 julio) 56-57.

García Marco, Francisco Javier (1998). Las bibliotecas y sus recursos humanos ante las nuevas tecnologías. // Asociación Andaluza de Bibliotecarios. Actas de las X Jornadas Bibliotecarias de Andalucía. Málaga: Asociación Andaluza de Bibliotecarios, 1998. 9-51.

Herrera Morillas, José Luis (2007). La imagen corporativa de las bibliotecas autonómicas españolas a través de Internet. // Scire: Representación y Organización del Conocimiento. 13:1 (en.-jun. 2007) 67-80.

Scire. $13: 1$ (en.-jun. 2007) 9-19. ISSN 1135-3716. 
Herrera Morillas, José Luis; Pérez Pulido, Margarita (2007). Análisis de la expresión información local en siete documentos de apoyo al desarrollo de las bibliotecas públicas. // Scire: Representación y Organización del Conocimiento. 13:1 (en.-jun. 2007) 121-132.

Pérez Pulido, Margarita; Herrera Morillas, José Luis (2007). Nuevos modelos organizativos en torno a la biblioteca pública y las bibliotecas de prisiones. // Scire: Representación y Organización del Conocimiento. 13:1 (en.-jun. 2007) 33-50.

Sánchez Hernández, María F. (2007). El acceso y la difusión de los recursos bibliográficos e informativos en la Biblioteca Regional de Extremadura. // Scire: Representación y Organización del Conocimiento. 13:1 (en.-jun. 2007) 81-86.

Suaiden, Emir José (2007). A dimensão social do conhecimento. // Scire: Representación y Organización del Conocimiento. 13:1 (en.-jun. 2007) 21-31.

Scire. $13: 1$ (en.-jun. 2007) 9-19. ISSN 1135-3716. 